NOTAS

\title{
Teoría de la productividad social
}

\author{
Theory of Social Productiviy
}

LEONARDO POLO

\section{Presentación}

\section{Introduction}

\author{
JUAN A. GARCÍA GONZÁLEZ \\ Universidad de Málaga
}

Este teXto de Leonardo Polo reproduce su intervención en las III Jornadas de filosofía de la política, organizadas por la cátedra de historia moderna y contemporánea de la filosofía de la Universidad de Málaga, y patrocinadas por las fundaciones Cánovas del Castillo y Hanns Seidel.

Se celebraron en el Centro de estudios de la fundación Hanns Seidel en Ojén (Marbella) del día 30 de enero al día 2 de febrero de 1985. Intervinieron en ellas los doctores José Luis del Barco, Ignacio Falgueras y Tomás Melendo (de la universidad de Málaga), Saturnino Álvarez Turienzo (de la universidad de Salamanca), Arturo Juncosa Carbonell (de la universidad central de Barcelona) y el propio Leonardo Polo Barrena (de la universidad de Navarra en Pamplona); el cual habló el sábado 2 de febrero a partir de las 12 horas.

La conferencia se grabó en cinta magnetofónica, y fue transcrita por Juan García; quien ha revisado el texto exclusivamente para adaptar el estilo coloquial de la conferencia al género escrito de un artículo de revista, el que aquí presentamos, y para establecer sus divisiones.

(C) Contrastes. Revista Internacional de Filosofía, vol. XXII-N² (2017), pp. 223-33. ISSN: 1136-4076

Departamento de Filosofía, Universidad de Málaga, Facultad de Filosofía y Letras Campus de Teatinos, E-29071 Málaga (España) 


\title{
Teoría de la productividad social
}

\section{Theory of Social Productiviy}

\author{
LEONARDO POLO \\ Madrid 1926 - † Pamplona, 2013
}

\section{RESUMEN}

En este texto se compara la filosofía política moderna, el absolutismo, especialmente de Hobbes, con el pensamiento sociológico contemporáneo, a partir de Adam Smith. Se ubica el cambio, o la diferencia, en la noción de productividad social. Para el pensamiento clásico la productividad social culmina en la idea de la virtud, más bien ausente en la sociología contemporánea. Aún por encima de la virtud Polo sugiere que está la noción de persona: su fecundidad es la raíz de la productividad social.

\section{PALABRAS CLAVE \\ ABSOLUTISMO, SOCIOLOGÍA, PRODUCTIVIDAD SOCIAL}

\section{ABSTRACT}

In this text we compare modern political philosophy, absolutism, especially Hobbes, with contemporary sociological thinking, from Adam Smith. The change, or the difference, is located in the notion of social productivity. For classical thought, social productivity culminates in the idea of virtue, rather absent in contemporary sociology. Above virtue Polo suggests that is the notion of person: its fecundity is the root of social productivity.

KEYWORDS

ABSOLUTISM, SOCIOLOGY, SOCIAL PRODUCTIVITY

EN LUGAR DE PARTIR DE LA FILOSOFÍA CLÁSICA, hoy voy a intentar partir de la filosofía política moderna.

Partiré de la siguiente tesis: la filosofía política moderna, en cuanto que tiene una adecuación suficiente con la situación política real, se puede dividir 
en dos fases. Una es la que, según mi consideración, se corresponde con el absolutismo, con el antiguo régimen tal y como a mí me parece más correcto entenderlo.

\section{EL ABSOLUTISMO}

Se suele decir que el antiguo régimen es la época europea que va desde la baja edad media hasta la revolución francesa; así lo entienden los historiadores, que incluyen buena parte de la edad media en el antiguo régimen. Pero la edad media no es con propiedad antiguo régimen, políticamente hablando; sino que por antiguo régimen hay que entender el despotismo o absolutismo monárquico.

El gran teórico, el que creo que da la talla exactamente, o el que se ajusta a esa situación política europea, es Hobbes.

Expondré esquemáticamente la filosofía política de Hobbes de la siguiente manera: Hobbes entiende que la relación del hombre con el hombre es una relación esencialmente conflictiva. A esto se suele llamar estado de naturaleza, y el mismo Hobbes emplea a veces esa expresión; pero yo preferiría denominarlo estado social prepolítico; porque en tanto en cuanto existe una relación entre hombres, lo social está ya en marcha. Sin embargo, ocurre que el dictamen que da Hobbes de las relaciones humanas en tanto que todavía no está construido el Leviatán, es simplemente el diagnóstico de una situación de conflictividad.

Dicho de otro modo, la tesis de Hobbes acerca de la sociedad en tanto que sociedad, al margen de la política que va a sobrevenir como remedio, es que no produce más que conflictos. La productividad social para Hobbes es conflictiva. Las relaciones humanas no producen nada que pueda estimarse positivo, y en este sentido, fuente de algo derivado valioso; sino que producen un profundo desvalor, una negatividad: la agresión como imposibilidad de establecer unas relaciones armónicas en sentido positivo. No hay ninguna armonía en las relaciones sociales y, por tanto, las relaciones sociales no son productivas sino de conflictos, o como conflicto.

Con esto, Hobbes está describiendo la desaparición del orden social medieval. O lo que es igual, está impresionado por el elemento experiencial histórico de las guerras religiosas. Este elemento experiencial le sirve de base para formular su postura. Es patente que las guerras religiosas, sobre todo la guerra de los treinta años, provocó en la conciencia europea una sensación profundamente desagradable y de sobresalto: ¿qué pasa aquí, que no nos entendemos? Estamos divididos por abismos, por posiciones contrapuestas; $\mathrm{y}$, por lo tanto, en cuanto intentamos el diálogo social, lo que se produce es la guerra de todos contra todos.

Además fue una guerra desastrosa; si se tiene en cuenta, por ejemplo, lo que le pasó a Alemania: que, después de esta guerra, quedó desolada, fuera de juego, arruinada por completo. Alemania se reparte según un criterio que abre 
paso al absolutismo; y que, para evitar la prolongación de la división civil en cada uno de los estados, estableció como principio que las circunscripciones territoriales se harían de acuerdo con el lema cuius regio eius religio. De tal modo que el monarca está dispuesto a expulsar, por el procedimiento que sea, a los disidentes religiosos; porque si hay disidencia religiosa se produce el homo homini lupus. Ésta es la base de la experiencia de Hobbes: un principio actuante en la política actual del momento.

Frente a la negatividad de la productividad de las relaciones humanas, Hobbes propone un remedio que consiste en la eliminación de la guerra, es decir, que el objetivo de Hobbes es exclusivamente la paz social. El Leviatán lo es por asumir en el poder político la entrega de las energías interhumanas, de esas energías que se crean en las relaciones, y lo que ellas producen, energía que globalmente es negativa. Y además el Leviatán es un ficto (en Hobbes hay una clara veta positivista que proviene de Ockham), pero que tiene cierta sustancia real; la cual consiste justamente en alimentarse con ese legado que es propiamente la iniciativa humana.

Es claro que esto da lugar a una desaparición de la teleología política. Cuando se entiende que la política es un añadido como remedio a la conflictividad social, entonces el objetivo político, o la capacidad teleológica de la política, se reduce al mínimo. Se ha señalado muchas veces que en Hobbes prácticamente no hay teleología. Y no sólo por ese mecanicismo básico suyo, que más bien es tardío: no algo de donde parte, sino que es traído más tarde como justificación de su pensamiento político. No es que Hobbes sea un mecanicista que aplica su mecanicismo a la política, sino que encuentra en el mecanicismo una feliz exclusión de la causa final que le conviene, y lo acepta. En efecto, el mecanicismo le viene muy bien para la interpretación de las relaciones humanas como estrictamente conflictivas: porque el mecanicismo produce choques. Sin embargo, Hobbes no conocía muy a fondo el mecanicismo.

En suma, nos encontramos con esto: que para Hobbes la política es el simple remedio a la productividad social, en tanto que la productividad social es negativa. Lo que, en último término, hace el estado es evitar esas consecuencias negativas estableciendo una especie de pacto, una situación que se constituye negativa, inversamente, respecto de la otra: si lo conflictivo es negativo, la paz será algo positivo. Pero, sin embargo, la situación de paz no es productiva. Esto es lo que tiene de incoherente el pensamiento de Hobbes; que, a su vez, es también el reflejo de lo que pasa en su momento histórico. Hobbes al Leviatán no le atribuye productividad política, sino que es un remedio político que consiste en la congelación de la productividad social.

Éste es el modelo que propongo para entender el pensamiento de Hobbes de una manera esquemática, y para entender lo que fue el absolutismo político. 
Hobbes no dice que el estado produzca. Hace algunas alusiones a que si hay paz, y teniendo en cuenta que el estado de guerra se continúa entre los estados, entonces al poder le interesará el fortalecimiento de sus individuos y el aprovechamiento de sus recursos, para estar en condiciones de afrontar la lucha a muerte que vuelve a aparecer en el orden internacional. Como se ve, esto no es un telos, propiamente dicho, el monarca no busca el bien de sus súbditos en un sentido progresivo, no busca el incremento de su prosperidad; lo único que al Leviatán le interesa es estar en disposición de poder luchar, de ser más fuerte que los otros Leviatanes. Es sólo por este motivo por el que el Leviatán, el poder absoluto, puede promover la industria y demás actividades sociales humanas.

Ésta es, a mi modo de ver, la primera fase del pensamiento político europeo, en cuanto que ese pensamiento se corresponde con la situación real, no en cuanto es una elucubración abstracta. Como elucubración, más que una utopía, sería una añoranza de otras épocas, es decir, una ucronía: un pensamiento político que aparece descolocado de las coordenadas de la situación política real del momento. Pero la situación política real es efectivamente la que se corresponde con la idea de que la productividad social se ha anulado, y la prueba de esta anulación son las guerras religiosas. Y, por lo tanto, no hay más solución que establecer un remedio, que es extrasocial: se impone desde fuera a la sociedad, y se justifica en cuanto que tal porque es el único remedio.

La política del remedio es una política que se aplica a la enfermedad social, es una terapéutica. Esta terapéutica no tiene carácter teleológico, sino que es preteleológica; porque si uno se reduce a la terapéutica, pierde los fines. Cuando algo se estropea hay que arreglarlo para que pueda funcionar; pero si se dice que sólo cabe arreglar, se ha perdido el fin: porque el arreglo no es más que una precondición del sistema para que funcione. Si se dice que no puede funcionar, y que sólo cabe remediar sus males, la noción de fin evidentemente se evapora. Esto ya lo observó Aristóteles cuando señalaba que la actitud del médico no es estrictamente teleológica.

\section{LA SOCIOLOGÍA}

La segunda fase del pensamiento político moderno se corresponde con la aparición de fuerzas sociales políticas, es decir, fuerzas positivas; a las cuales no se les puede atribuir la índole de la productividad, la índole social productiva, en los términos hobbesianos, negativos. Los pensadores que se adecúan con esta nueva situación, ya lejana de las guerras religiosas y en la que van apareciendo movimientos, grupos sociales, dispuestos a generar una productividad social positiva, es decir, no conflictiva, serán aquéllos que, más que poner el énfasis en lo político como remedio de lo social, se fijan en el carácter productivo de la sociedad y hacen entonces sociología. Estrictamente, la sociología -a mi modo 
de ver- tiene su momento fundacional, como punto de vista que pretende un carácter científico pero diferencial, en el mismo momento en que se sienta la tesis de que existe productividad social positiva.

El tratamiento filosófico o científico de la política se centra entonces en la sociedad definida como producción: la sociedad es producción mientras que el monarca absoluto es improductivo; se han cambiado las tornas. Pero, por otra parte, es un cambio muy sencillo, a saber: la descalificación del poder absoluto se basa en su improductividad, contrastada con la percepción neta de una productividad positiva en la sociedad. Esto es, insisto, sociología. Dicho de otro modo: el concepto central, sin el cual no se constituye la sociología como ciencia suficientemente diferenciada, es el concepto de producción; si la sociedad no produce no tiene sentido considerarla. Que haya una ciencia que se base en lo social, es posible porque lo social es producción positiva.

Cuando se consulta a los sociólogos profesionales, lo que a uno le dicen es esto: que la categoría central es la de producción social. La sociedad se explica desde el punto de vista de su productividad. Los problemas estructurales responden después a la necesidad de un incremento de productividad, pero el concepto fundamental es éste último. Por eso, me atrevería a decir que la sociología como pensamiento político, que se hace sociológico en el primer tercio del XIX, no es una fase más entre épocas, sino más bien el intento de fundar una época: la época de la producción social, la época basada en la actuosidad, en la fecundidad de la acción social. La actividad social se considera desde la formalidad de la productividad; es decir, las actividades son sociales en tanto que productivas, y si no, no son sociales.

En esta línea se mueven todos los grandes pensadores políticos, de un signo o de otro. Hacer un elenco de los que considero más relevantes, o estrictamente adecuados con el cambio de signo (de la consideración de lo político como tal, a la consideración de lo social como tal, en virtud de que la productividad social es central, mientras que la productividad política es nula), sería mencionar a pensadores de signo muy diferente. Como por ejemplo un tradicionalista, De Bonald. Todo su pensamiento político es un pensamiento sociológico productivo, y su preocupación central es encontrar la lógica de esa productividad, es decir, el logos social. De Bonald considera que es una equivocación la aplicación de los modelos del siglo XVIII, de racionalidad ilustrada, porque con esos modelos no se consigue productividad social. Esos modelos racionales no son la razón social; pero, bien entendido que razón social significa estrictamente razón práctica, y que razón práctica en este contexto significa razón productiva social. La productividad social es lo descubierto, no por una elucubración teórica, sino porque existen de hecho las fuerzas sociales productivas.

Esta tesis está en De Bonald; pero también está en Hegel, aunque Hegel pasa por dos fases. No voy a analizar a Hegel, porque sería muy largo; pero eso 
está presente tanto en la Fenomenología del espíritu, como en la Filosofía del derecho, aunque ahora, ya en Berlín, el tratamiento es diferente. Igualmente, Comte es un sociólogo, y Saint Simon también. Ya no es un pensamiento político en sentido moderno; porque un pensador político en sentido moderno es el que piensa en el poder como algo extrínseco a la sociedad, sobre la base de la negatividad o improductividad social.

\section{BALANCE ACTUAL}

Sentado esto, hay que señalar ahora dos cosas.

Primero: ¿se puede mantener hoy en día la idea de productividad social? Dicho rápidamente: ¿hoy estamos en una fase comtiana o en una fase hobbesiana?, ¿ha hecho crisis la idea de productividad social?, ¿consideramos hoy que la productividad social es profundamente más conflictiva que lo que antes se pensaba? Ya no es lo que decía Hobbes de la guerra de todos contra todos, sino que es la bomba atómica, la destrucción de todos; o es la técnica produciendo la destrucción del planeta, la crítica ecologista, etc. La tesis ecologista es hobbesiana, es decir, piensa que la productividad humana es negativa en orden al planeta, al cosmos. Cósmicamente la sociedad es una excepción a la homeostasis, pues destruye toda homeostasis. Ésta es, pues, la primera pregunta que uno se tiene que plantear. ¿Estamos hoy dispuestos a ser hobbesianos, y a pensar que tiene que haber un remedio extrínseco a una conflictividad social constitutiva, es decir, a una productividad social negativa?; o más bien estamos dispuestos a seguir admitiendo la noción de productividad social. Ése es el asunto.

En el pensamiento de Carlos Marx (que es fundamentalmente un sociólogo) es evidente también que la noción de producción es central. Lo que ocurre en Marx es que la noción de producción está aplicada, pensada, de una manera en cierto modo poco crítica e insuficiente; porque además de ser la suya una crítica bastante superficial, la noción de producción está un poco descolocada. Marx empieza a ver la noción de producción hacia 1860, en Bruselas, y a mi modo de ver su concepción está desfasada, es un poco ucrónica, y por lo tanto sólo puede ser asumida críticamente. Ésta es la fundamental ambigüedad del pensamiento marxiano. El pensamiento de Marx lo mismo puede ser una teoría de la productividad social elevada al máximo en un cierto nivel, es decir, la sociedad perfecta sería aquélla en la que la productividad social es suficiente, está equilibrada; o bien puede ser tomado en la línea de Lenin, a saber: sosteniendo la tesis de que no hay productividad social. Esto es muy claro: Lenin es Hobbes. Esta ambigüedad es debida a que la teoría de la productividad social en Marx tiene un elemento crítico insuficiente.

Ahora bien, si tenemos en cuenta aquella pregunta, que es estrictamente necesario hacérsela hoy, es decir, la de si apostamos o no por la productividad 
social, entonces, para poder responderla, hay que examinar el significado estricto de la productividad social. Porque evidentemente la productividad social es un concepto (y aquí es donde aparece la necesidad de la crítica y la disparidad de opiniones) sumamente plurivalente. El conflicto que ocasiona esta plurivalencia en la concepción de la productividad social, a mi modo de ver, es debido a que lo que estos hombres descubrieron es que efectivamente hay productividad social; y en este sentido restauraban el pensamiento clásico, que está basado también en la tesis de la productividad social.

Segundo: en el pensamiento clásico la productividad se centra fundamentalmente en la virtud. La sociedad genera virtudes o vicios entre los hombres, en tanto en cuanto los hombres se relacionan. En la sociología moderna se vuelve a tomar contacto con la noción de producción; es un darse cuenta de nuevo de que la productividad social es importantísima. Pero la cuestión es ahora examinar en qué consiste la productividad social. Insisto en que la productividad social consiste en la virtud. Esto es precisamente lo que se excluye en la sociología moderna; ésta es la diferencia: que la sociedad ahora no produce virtud, sino otras cosas.

La ética social utópica aspira a lo que sea menos a la virtud, y eso porque la utopía es una teoría de la productividad social mecánica. Hay productividad social, puesto que vamos de una situación a otra mejor; pero ¿cómo se logra la situación mejor?. Y, por otro lado, ¿en la situación mejor, el hombre es mejor?. Para ilustrar esto acudiré a la parábola evangélica de los invitados a las bodas. Recuerden que el Evangelio dice que todos fueron invitados a las bodas, pero que unos no tenían el vestido adecuado, y fueron echados fuera sin contemplaciones. Pues la utopía es aquella interpretación del final feliz como consecuencia de una productividad automática, en que los convidados no precisan traje de bodas, es decir, no tienen virtudes. Es una sociedad magnífica hecha de mediocres, una sociedad perfeccionada sin perfeccionamiento propio; la productividad social se ha visto en términos de resultados, no en términos cibernéticos, de retroalimentación, es decir, en términos de plantearse la cuestión de qué le pasa al hombre cuando produce otras cosas, qué tipo de producción tiene lugar en el hombre cuando actúa. Ésa es una omisión gravísima, es la omisión del tema de la virtud.

Si la productividad social se entiende en un sentido pleno, hay que decir que cualquier mejora que el hombre consiga actuando comunitariamente hay que considerarla en último término en el orden de la naturaleza humana misma, sin que ello excluya su consideración en el orden de la productividad. En el orden de la naturaleza humana lo estrictamente productivo, la más alta potenciación de la capacidad productiva humana, es la producción de la virtud. Si eso se omite, ya lo dijo san Agustín: resulta que hay hombres que se dedican a construir instrumentos perfectísimos, pero que se envilecen al 
hacerlos. Pagar el durísimo tributo del envilecimiento humano a costa de otro tipo de productividad es una visión reductiva de la productividad social. Aquí los clásicos siguen mandando, son estrictamente actuales; si se entiende que la pregunta por la productividad humana busca una respuesta integral acerca de la productividad.

Sin embargo, yo diría algo más. Me parece que la virtud tampoco es la última palabra. Con todo, me parece que el pensamiento clásico es estrictamente actual, porque la virtud es insustituible: si no se producen virtudes, se produce el envilecimiento; y entonces todas las demás producciones sociales se vienen abajo. Sin embargo, yo no considero por eso que la época moderna sea una decadencia respecto de la época clásica. No es eso; sino que todos los descubrimientos de productividad que se logran en la época moderna están intrínsecamente amenazados por un error constitutivo: el olvido de la virtud; porque, cuando se olvida la virtud, la productividad social deviene una productividad entrópica, entra en pérdida y no hay quien la pare. Una ética sin virtudes no es una ética; y sobre todo no es una ética social, esto es, una ética para los ciudadanos en cuanto que los ciudadanos producen.

La gran caracterización de la ética aristotélica respecto de los ciudadanos es que éstos son productivos, y por eso las relaciones entre los ciudadanos son políticas y no despóticas. La distinción entre ambas, y el poner el poder despótico en el dominio sobre las cosas, es una adquisición frente a Platón de primer orden; y está establecida en términos de productividad. Política significa aquel tipo de orden que se dirige a un agente libre, que exige una ejecución ulterior a la orden. En cambio, en el poder despótico, la orden con su contenido instructivo se aplica a algo inerte, es decir, produce la obra sin más; de tal manera que la obra no se tiene que encargar de ninguna ejecución posterior. La relación cuerpo-alma en Aristóteles es despótica; pero una relación entre iniciativas libres, o entre ordenadores y ejecutores, no puede ser despótica sino política.

Ésta es entonces la cuestión: ¿producimos... y nos desproducimos? Hoy esto está muy de moda, porque naturalmente ha calado, y nosotros somos herederos de los descubridores de la sociología. Por ejemplo, hablamos de autorrealización: la autorrealización es una teoría de la producción, es una modalidad de producción. Si piensan en los grandes autores del siglo XIX verán que todos ellos se centran en la producción, aunque sus interpretaciones de la producción sean más o menos desfasadas, más o menos afectadas por la crítica, y con una visión parcial de la producción. O bien están extrasocializadas por recabamiento de otra instancia, como es aquello a lo que Nietzsche llama la vida. La filosofía de Nietzsche es una pura filosofía de la producción; pero no es sociología porque para Nietzsche la sociedad propiamente hablando no produce. Pero, sea como sea, la noción de producción está presente.

Finalmente, voy a establecer para acabar dos tesis: 
Primera. La sociología del siglo XIX no consiguió establecer el concepto de productividad social en su contenido mismo. Se dio cuenta de que existe la productividad social; sin embargo no pudo responder jamás a qué es la productividad social. Lo que se tradujo en la introducción de un elemento utópico. Lo utópico no es marxista ni es algo exclusivo de esta línea de pensamiento. El primer utópico fue Adam Smith.

Adam Smith dice lo siguiente: la productividad del individuo no es social, porque el individuo siempre que produce actúa movido por la avaricia y el egoísmo. Precisamente porque ésa es la causa de su productividad, si el individuo intentara ser benéfico, entraría en contradicción con la propia índole de su productividad, y por lo tanto se transformaría automáticamente en improductivo. Y sin embargo, dice, no puede admitirse que la productividad individual no sea a su vez social. Pero ¿cómo lo es? Pues por un procedimiento en definitiva mecánico, que él llama «la mano invisible». La mano invisible es un elemento utópico puro, puesto que la utopía consiste en decir que la productividad social es automática, es decir, extrahumana; en eso consiste su sociología. Leo el texto: «el empresario no pretende promover el bienestar público ni sabe cuándo está promoviéndolo. Al dirigir su industria de tal manera que el producto alcance su máximo valor, sólo busca su propio beneficio; pero le guía una mano invisible para contribuir a un objetivo que no formaba parte de lo que perseguía».

Esta articulación de Adam Smith no ha sido abandonada. Me atrevería a decir que no hay ningún sociólogo que haya acertado a replicar a Adam Smith. Hay un elemento social, y por ahí se va a la inmortalización de la sociedad, estrictamente extrahumano; y por tanto definido como social por exclusión del agente humano. Elemento que justamente está en estricta incoherencia con la acción humana, pero es así como consigue la producción social.

Y hay que decir: ¡vaya una manera de formular en un cuerpo teórico el descubrimiento de la productividad social! Adam Smith está convencido de que hay productividad social; pero cuando se trata de decir cómo se produce, se encuentra con un prejuicio: el prejuicio luterano de que el hombre es malo por naturaleza; y por tanto no cabe proponer que el hombre pueda hacer algo bueno, y tiene que recurrir a la mano invisible. Entonces la sociedad se separa del individuo, y al separarse aparece esa especie de quasi Leviatán, etc. Todo esto está implícito en la mala interpretación de la productividad social de Adam Smith, que es una utopía.

Por otro lado, esta concepción está refrendada por la crítica de Marx, porque Marx acepta de modo íntegro la tesis de Adam Smith, y se basa en ella. La noción de productividad de Carlos Marx es sumamente reducida, porque para Marx el hombre es el productor sólo de las condiciones de su existencia física. Y, equívocamente, desde ahí el hombre es productor de una ficción que se llama superestructura. Definir la productividad humana como la produc- 
tividad de las condiciones de posibilidad de la propia existencia física es una teoría superficial de la productividad; además de extremadamente reducida, porque las otras son pseudoproducciones, superestructuras.

\section{LA PERSONA}

Segunda y última tesis. Yo diría que, en último término, la actividad característica de la sociedad es, desde luego, la virtud; pero también su opuesto, y aquí entra un tema que es la eventual depravación del hombre, de la naturaleza humana; con todo, detrás de ambas, bajo la virtud y el envilecimiento, aún está la persona. Lo diré de la siguiente manera, recordando a un profesor de derecho de la universidad de Santiago que una vez dijo la siguiente frase: «voy a dividir este curso en tres mitades»; naturalmente esto ha pasado a la historia como ejemplo de una estupidez. Pero dándole vueltas al asunto, creo que la productividad social consiste justamente en que haya esas tres mitades: el amejoramiento o estropicio del hombre, y por detrás la fecundidad de la persona; ésta es la más alta y la más propia forma de productividad social. 
